\title{
An Analysis of two Sustainable projects in the light of the LEED-NC and LEED-ND rating systems
}

\author{
F. Roseta-Vaz-Monteiro ${ }^{1, *}$, E. M. Karayianni-Vasconcelos ${ }^{1}$ \\ ${ }^{1}$ CIAUD - Faculty of Architecture, Technical University of Lisbon, Lisbon, Portugal \\ *Tel: +351 916746476, E-mail: froseta@fa.utl.pt (or) filipa.roseta@network.rca.ac.uk
}

\begin{abstract}
The methodology used in this paper consists in studying how the 5 environmental categories of the LEED New Constructions and Major Renovation plus the 3 categories of the LEED Neighbourhood Development apply to the architectural and urban design strategies of two projects: Masdar City and "Forwarding Dallas". We chose two very different but at the same time similar projects which claim to be environmentally conscious. On one end, there is the self-intituled "world's first carbon-neutral city", Masdar, designed by Foster + Partners and one of the largest and most ambitious developments of its kind. On the opposite end, and in a significantly smaller scale, there is the "Re-Vision Dallas" competition winner project, "Forwarding Dallas", designed by a collaboration of two Portuguese architecture practices: DATA + MOOV. This competition promoted the idea of transforming a vacant inner-city block in Dallas into a carbon-neutral neighbourhood, creating, for that purpose, a prototype for an innovative, sustainable urban community.
\end{abstract}

This paper strives to highlight, through the comparative analysis of these two projects, how the desire to meet high standards of sustainability not only affects the practice of architecture and urban design, but might also generate a particular architectural language with identifiable physical characteristics.

Keywords: Carbon-Neutral, Masdar City, Forwarding Dallas, LEED, Renewable Energy

\section{Introduction}

This paper presents part of a research developed by a research unit, named " $+E-C O 2$ ", or "More Energy, Less Carbon", based in Lisbon, at Faculdade de Arquitectura da Universidade Técnica de Lisboa, which pursues the goal of understanding how the carbon neutral challenge is changing present day's urban design and architectural practices. Our purpose is to focus on the design process in order to assess two key questions: 1) How does the desire to meet high standards of sustainability affect the practice of architecture and urban design? 2) Will this global concern with a carbon-neutral future generate a particular architectural language with identifiable physical characteristics regardless of location?

\section{Methodology}

We have determined as research methodology for our $+E-C O 2$ research project the analysis of self-acclaimed carbon neutral and/or carbon reduction projects in order to detect how the carbon neutral challenge is affecting the practice of urban and architectural design. The projects which claim to be carbon neutral are the most relevant for our research because, in these projects, the practitioners are guided by the clear purpose of configuring architecture and urban space so as to meet the carbon neutral challenge.

Our analysis derives from the comparative process of design strategies in light of fixed criteria, enabling us to highlight specific design differences and/or resemblances in between the projects. For this paper, we have chosen as fixed criteria the 5 environmental categories of the LEED New Constructions and Major Renovation [1] plus the 3 categories of the LEED Neighborhood Development [2]. In light of these 8 categories, we analyze and compare two very ambitious projects in what regards the carbon neutral challenge. 
The first project is the development of a block in Dallas designed by a collaboration of two Portuguese architectural practices (MOOV+DATA), which obtained the first prize in an international architectural competition promoted by the American non-profit organization named "Re-vision". The competition's brief challenged architects to design a project able to become LEED Platinum. Most information regarding the project has been provided by António Louro, one of the architects/partners managing the project [3]. The project's brief was to redesign an urban block in Dallas, including mostly housing, but also commercial spaces and urban equipments.

The second project is the Masdar City development designed by Foster+Partners which aims to become the first carbon neutral city to ever be built. The Masdar City development is located in Abu Dhabi, close to the international airport and was conceived to be carbon neutral, zero-waste, car-free city for 40,000 residents and 50,000 daily commuters. The city is designed for an area of six million square meters. Even if the city is currently under construction (the completion of the first building was announced in November 2010), its scale and ambitious program as a research and institutional hub dedicated to Renewable Energies make it a most relevant project to follow, as noted by Reiche [4]. Data cited in this paper has been gathered from other papers, from the information provided by the company responsible for the development, the Abu Dabhi Future Energy Company [5], by the architects responsible for the masterplan at Foster + Partners [6], and by articles included in architectural magazines [7]. Papers reviewed regarding Masdar City relate to specific technological innovations in the energy field [8], to policies supporting the development [4] or to specific buildings [9]. Prior to this paper, we have presented a paper on the possibility of using some of Masdar City's renewable energy strategies in a "bottom-up" type of development [10]. For the current paper, our approach is different as we aim to highlight the impact of the carbon neutral challenge on architectural and urban design.

We have determined as primary data the information coming from the practitioners or from the entities commissioning the projects. This data must be taken as a set of design guidelines which might not be possible to complete or might change with the project's development (as it has already happened in the Masdar City development with the experimental transport system, originally elevated and currently underground).

We chose to use the LEED categories as fixed criteria due to this rating system's holistic approach and to the system's ability of evaluating projects worldwide; however, unlike the LEED rating system, which aims to provide a quantifiable benchmark to compare each project's efficiency, we aim to assess the impact of the carbon neutral challenge on architectural design; hence, some categories might have no effect on architecture (such as the efficiency of appliances), while others will change the configuration of the city of the future. In Table 1, we present the relationship we created in between the LEED categories and the architectural and urban design strategies of the two case-studies selected. We created a rating system measuring the Impact on Architectural Design (IAD), presenting five classes ranging from IAD 0 to IAD4 where: IAD 0 classifies the strategy as having no or low direct impact on architectural design; IAD 1 classifies the strategy as affecting architectural surfaces using existing design strategies; IAD 2 classifies the strategy as affecting architectural surfaces using new technologies and/or innovative design strategies; IAD 3 classifies the strategy as affecting architectural form using existing design strategies; and IAD 4 classifies the strategy as affecting architectural form using new technologies and/or innovative design strategies. 
Table 1. Relationship between LEED categories and architectural and urban design strategies

LEED Environ. Categories

(NC=NewConstruction; $\mathrm{ND}=$

Neighborhood Development)

The categories which, on

both case-studies, did not

apply or had NINF were

excluded for this paper

\begin{tabular}{|c|c|c|}
\hline & Forwarding Dallas & Masdar City \\
\hline \multicolumn{3}{|l|}{ NC - Sustainable Sites } \\
\hline $\begin{array}{l}\text { Construction Activity } \\
\text { Pollution Prevention }\end{array}$ & IAD 0 & $\begin{array}{l}\text { Impacts assessed + mitigation } \\
\text { actions predicted [10] IAD } 0\end{array}$ \\
\hline Site Selection & $\begin{array}{c}\text { Block within built } \\
\text { neighbourhood. IAD } 3\end{array}$ & $\begin{array}{c}\text { 6km, built mostly on former } \\
\text { plantations land. IAD } 3\end{array}$ \\
\hline $\begin{array}{l}\text { Development Density and } \\
\text { Community Connectivity }\end{array}$ & $\begin{array}{l}\text { Optimizes pedestrian public } \\
\text { space + connectivity public } \\
\text { space for pedestrians+ } \\
\text { bicycles inside the block. } \\
\text { IAD } 3\end{array}$ & $\begin{array}{l}\text { Optimizes pedestrian public } \\
\text { space + connectivity public } \\
\text { space for pedestrians+ } \\
\text { bicycles inside the block. } \\
\text { IAD } 3\end{array}$ \\
\hline Alternative Transportation & $\begin{array}{l}\text { No public transport system } \\
\text { available. Promotes cyclable } \\
\text { public spaces, car-pooling } \\
\text { and bicycle parking. IAD } 3\end{array}$ & $\begin{array}{l}\text { Car-free city. New transport } \\
\text { system, } 2 \text { levels: LRT } \\
\text { (aboveground), PRT } \\
\text { (undergound). IAD } 4\end{array}$ \\
\hline Site Development & $\begin{array}{l}\text { Use of native vegetation in } \\
\text { public spaces and rooftops. } \\
\text { IAD } 4\end{array}$ & $\begin{array}{l}\text { Compact footprint and } \\
\text { limited, or walled, perimeter. } \\
\text { IAD } 3\end{array}$ \\
\hline Stormwater Design & $\begin{array}{c}\text { includes vegetative roofs and } \\
\text { pervious pavements IAD } 4\end{array}$ & NINF \\
\hline Heat Island Effect & $\begin{array}{c}\text { Roofs covered with } \\
\text { vegetative surfaces. IAD } 4\end{array}$ & $\begin{array}{c}\text { Roofs with shading structures } \\
\text { covered by photovoltaic } \\
\text { panels. IAD } 4\end{array}$ \\
\hline
\end{tabular}

LEED NC - Water

Efficiency (WE)

Water Use Reduction

\section{in agriculture + graywater \\ used in toilets IAD 4}

Rainwater collected and used
Architectural and Urban Design Strategies

IAD Impact on architectural design 0-4))

\begin{tabular}{ccc}
\hline Water Use Reduction & $\begin{array}{c}\text { Rainwater collected and used } \\
\text { in agriculture + graywater } \\
\text { used in toilets IAD 4 } \\
\text { Water Efficient Landscaping }\end{array}$ & $\begin{array}{c}\text { Rainwater collected } \\
\text { +graywater used in toilets } \\
\text { and cooling of public spaces. } \\
\text { IAD 4 }\end{array}$ \\
\hline $\begin{array}{c}\text { Native plant use + drop-by- } \\
\text { drop irrigation system + on } \\
\text { site measurements to assess } \\
\text { irrigation needs. IAD 4 }\end{array}$ & $\begin{array}{c}\text { Native Plant use+ Intelligent } \\
\text { irrigation systems. IAD 4 } \\
\text { Technologies }\end{array}$ & $\begin{array}{c}\text { On-site waste water treatment } \\
\text { by gravity-driven mechanical } \\
\text { systems + sand and UV light } \\
\text { filtration. IAD 4 }\end{array}$ \\
\hline Water Use Reduction & NINF & NINF \\
\hline NC -Energy and Atmosphere & & $\begin{array}{c}\text { Initial use of desalinized } \\
\text { water and recycling of most } \\
\text { water in the system. IAD 3 }\end{array}$ \\
\hline OptimizeEnergy Performance & High energy performing & NINF \\
\hline
\end{tabular}




\begin{tabular}{|c|c|c|}
\hline \multicolumn{3}{|c|}{$\begin{array}{c}\text { appliances + LED technology } \\
\text { IAD } 0\end{array}$} \\
\hline On-site Renewable Energy & $\begin{array}{c}\text { On the rooftop - Solar } \\
\text { thermal energy + } \\
\text { photovoltaic + wind energy. } \\
\text { Roof design expands surface } \\
\text { to maximize production. } \\
\text { Solar cells on the façade. } \\
\text { IAD } 4\end{array}$ & $\begin{array}{l}\text { On the rooftop - almost } 80 \% \\
\text { of the consumed energy will } \\
\text { be solar. The entire city } \\
\text { covered with panels. IAD } 4\end{array}$ \\
\hline Enhanced Commissioning & $\begin{array}{l}\text { Specialized consultants } \\
\text { included. IAD } 0\end{array}$ & $\begin{array}{l}\text { Specialized consultants } \\
\text { included. IAD } 0\end{array}$ \\
\hline $\begin{array}{l}\text { Enhanced Refrigerant } \\
\text { Management }\end{array}$ & $\begin{array}{l}\text { Non-mechanical methods of } \\
\text { ventilation (cross-ventilation } \\
\text { in every apartment). IAD } 3\end{array}$ & $\begin{array}{c}\text { Traditional methods of } \\
\text { cooling (such as cooling } \\
\text { chimneys) + mechanical } \\
\text { cooling. IAD } 3\end{array}$ \\
\hline Measurement/ Verification & IAD 0 & IAD 0 \\
\hline Green Power & IAD 0 & IAD 0 \\
\hline \multicolumn{3}{|l|}{$\begin{array}{c}\text { NC - Materials and } \\
\text { Resources }\end{array}$} \\
\hline $\begin{array}{c}\text { Storage and Collection of } \\
\text { Recyclables }\end{array}$ & $\begin{array}{c}\text { Includes area for Storage and } \\
\text { Collection of Recyclables. } \\
\text { IAD } 0\end{array}$ & $\begin{array}{c}50 \% \text { waste will be recyclable, } \\
17 \% \text { will become fertilizers } \\
\text { and } 33 \% \text { will not be } \\
\text { recyclable. IAD 0 }\end{array}$ \\
\hline $\begin{array}{l}\text { Construction Waste } \\
\text { Management }\end{array}$ & $\begin{array}{l}\text { 100\% pre-fabricated building } \\
\text { systems to reduce on site } \\
\text { impact and waste. IAD } 2 \\
\end{array}$ & NINF \\
\hline Recycled Content & Recycled Wood. IAD 2 & $\begin{array}{c}\text { Materials with a } 30 \% \\
\text { recycled content predicted. } \\
\text { IAD } 2\end{array}$ \\
\hline Regional Materials & $\begin{array}{c}\text { Use of locally produced } \\
\text { materials. IAD } 1\end{array}$ & NINF \\
\hline \multicolumn{3}{|l|}{ NC-Indoor Envirn. Quality } \\
\hline $\begin{array}{l}\text { Minimum Indoor Air Quality } \\
\text { Performance Required }\end{array}$ & $\begin{array}{l}\text { Non-mechanical methods of } \\
\text { ventilation (cross-ventilation } \\
\text { in every apartment). IAD } 3\end{array}$ & $\begin{array}{l}\text { Traditional methods of } \\
\text { cooling (such as cooling } \\
\text { chimneys) + mechanical } \\
\text { cooling. IAD } 3 \\
\end{array}$ \\
\hline $\begin{array}{c}\text { Environmental Tobacco } \\
\text { Smoke- Control Required }\end{array}$ & IAD 0 & IAD 0 \\
\hline $\begin{array}{c}\text { Outdoor Air Delivery } \\
\text { Monitoring }\end{array}$ & IAD 0 & IAD 0 \\
\hline $\begin{array}{c}\text { Controllability of Systems- } \\
\text { Lighting and Thermal } \\
\text { Comfort }\end{array}$ & $\begin{array}{c}\text { Operable Windows and } \\
\text { Window Protections IAD } 2\end{array}$ & NINF \\
\hline $\begin{array}{l}\text { Thermal Comfort- } \\
\text { Design/Verification }\end{array}$ & $\begin{array}{l}\text { Northeast façades in high } \\
\text { thermal mass straw walls. } \\
\text { IAD } 2\end{array}$ & NINF \\
\hline Daylight and Views & $\begin{array}{l}\text { Block designed to maximize } \\
\text { daylight and views. Windows } \\
\text { consider southern orientation } \\
\text { and permanent shading }\end{array}$ & NINF \\
\hline
\end{tabular}


devices. IAD 3

\begin{tabular}{|c|c|c|}
\hline \multicolumn{3}{|c|}{ devices. IAD 3} \\
\hline $\begin{array}{l}\text { NC/ND -Innovation in } \\
\text { Design }\end{array}$ & & \\
\hline Innovation in Design & Educational project IAD 0 & Educational project IAD 0 \\
\hline $\begin{array}{l}\text { LEED Accredited } \\
\text { Professional }\end{array}$ & $\begin{array}{l}\text { Goal to obtain } \text { LEED } \\
\text { Platinum IAD } 0\end{array}$ & NINF \\
\hline \multicolumn{3}{|l|}{$\begin{array}{l}\text { NC/ND-Smart Location and } \\
\text { Linkage }\end{array}$} \\
\hline Smart Location & $\begin{array}{c}\text { Block within existing } \\
\text { neighborhood in Dallas. } \\
\text { IAD } 3\end{array}$ & $\begin{array}{c}\text { New development on } \\
\text { previously planted land close } \\
\text { to national transport } \\
\text { infrastructure (airport). Max. } \\
\text { walkable distance from the } \\
\text { planned PRT stops is } 150 \mathrm{~m} \text {. } \\
\text { IAD } 4 \\
\end{array}$ \\
\hline $\begin{array}{l}\text { Imperiled Species and } \\
\text { Ecological Communities }\end{array}$ & NA & $\begin{array}{c}\text { Loss of existing habitats } \\
\text { predicted. Mitigation actions } \\
\text { predicted. IAD } 3\end{array}$ \\
\hline $\begin{array}{l}\text { Wetland and Water Body } \\
\text { Conservation }\end{array}$ & NA & $\begin{array}{c}\text { No significant effects } \\
\text { predicted in the initial stages. } \\
\text { IAD } 0\end{array}$ \\
\hline $\begin{array}{l}\text { Agricultural Land } \\
\text { Conservation }\end{array}$ & NA & $\begin{array}{c}\text { Located predominantly on } \\
\text { abandoned plantations. IAD } 3\end{array}$ \\
\hline Preferred Locations & Existing City. IAD 3 & $\begin{array}{c}\text { Located predominately on } \\
\text { abandoned plantations - new } \\
\text { infrastructures considered. } \\
\text { IAD } 3\end{array}$ \\
\hline $\begin{array}{l}\text { Locations with Reduced } \\
\text { Automobile Dependence }\end{array}$ & $\begin{array}{l}\text { Included in city with high } \\
\text { automobile dependence. } \\
\text { IAD3 }\end{array}$ & Car-free city. IAD 4 \\
\hline Bicycle Network and Storage & Predicted. IAD 3 & $\begin{array}{c}\text { Mostly walkable distances. } \\
\text { IAD } 3\end{array}$ \\
\hline Housing and Jobs Proximity & $\begin{array}{l}\text { Mostly residential (dwellings } \\
\text { and support areas = } 303658 \\
\text { sq.f; commercial, equipments } \\
\text { and greenhouses } 101828 \text { sq. } \\
\text { f, parking } 68867 \text { sq. f). IAD } 3\end{array}$ & $\begin{array}{l}\text { Mixed-use development } \\
\text { (30\% residential; } 20 \% \text { tax- } \\
\text { free enterprises; } 10 \% \\
\text { commercial services; } 3 \% \\
\text { cultural equipments, } \\
\text { 3\%university , 34\% with } \\
\text { parking, services, recreational } \\
\text { spaces, and other areas). } \\
\text { IAD3 } \\
\end{array}$ \\
\hline $\begin{array}{l}\text { Site Design for Habitat or } \\
\text { Wetland and Water Body } \\
\text { Conservation }\end{array}$ & NA & $\begin{array}{l}\text { Loss of existing habitats } \\
\text { predicted. Mitigation actions: } \\
\text { creation of new habitats with } \\
\text { native species through the } \\
\text { greenfingers + retention of } \\
\text { desert habitat outside } \\
\text { citywalls + healthy native } \\
\text { plant specimens translocated } \\
\text { to Masdar's nursery. IAD } 3\end{array}$ \\
\hline
\end{tabular}




\begin{tabular}{|c|c|c|}
\hline $\begin{array}{l}\text { Restoration + Long Term } \\
\text { Conservation Management of } \\
\text { Habitat or Wetlands and } \\
\text { Water Bodies }\end{array}$ & NA & $\begin{array}{l}\text { Mitigation actions predicted. } \\
\text { IAD } 3\end{array}$ \\
\hline \multicolumn{3}{|l|}{$\begin{array}{l}\text { ND-Neighborhood Pattern + } \\
\text { Design }\end{array}$} \\
\hline Walkable Streets & $\begin{array}{l}\text { Pedestrian space within the } \\
\text { block. Ground level with } \\
\text { commercial and other } \\
\text { equipments with glass } \\
\text { façades. IAD 3 }\end{array}$ & $\begin{array}{l}\text { Car-free city. Most of the } \\
\text { transport system is } \\
\text { underground to free public } \\
\text { space for pedestrians. IAD } 4\end{array}$ \\
\hline Compact Development & Increases footprint IAD 3 & $\begin{array}{l}\text { Compact footprint with rigid } \\
\text { physical limit perimeter to } \\
\text { prohibit urban sprawling. } \\
\text { IAD } 3\end{array}$ \\
\hline $\begin{array}{l}\text { Connected and Open } \\
\text { Community }\end{array}$ & $\begin{array}{l}\text { Block designed (sectioned) to } \\
\text { increase existing } \\
\text { connectivity. IAD } 3\end{array}$ & $\begin{array}{l}\text { Requirement to place every } \\
\text { pedestrian at a distance of } \\
\text { 150m from a PRT station in } \\
\text { any given location increases } \\
\text { connectivity. IAD } 4\end{array}$ \\
\hline $\begin{array}{l}\text { Mixed-Use Neighborhood } \\
\text { Centers }\end{array}$ & NA & $\begin{array}{l}\text { Mixed-use. Centers related to } \\
\text { transit system. IAD } 3\end{array}$ \\
\hline $\begin{array}{c}\text { Mixed-Income Diverse } \\
\text { Communities }\end{array}$ & $\begin{array}{c}\text { Dwellings range from studio } \\
\text { to } 3 \text { bedroom flats IAD } 3\end{array}$ & NINF \\
\hline Reduced Parking Footprint & $\begin{array}{c}\text { Reduced surface parking } \\
\text { IAD } 3\end{array}$ & $\begin{array}{l}\text { Parking outside city walls } \\
\text { IAD } 3\end{array}$ \\
\hline Street Network & $\begin{array}{c}\text { Block designed (sectioned) to } \\
\text { increase existing } \\
\text { connectivity. IAD } 3 \\
\end{array}$ & $\begin{array}{l}\text { Every pedestrian at a distance } \\
\text { of } 150 \mathrm{~m} \text { from a PRT station } \\
\text { in any given location. IAD } 4\end{array}$ \\
\hline Transit Facilities & $\begin{array}{c}\text { Promotes cyclable public } \\
\text { spaces, car pooling and } \\
\text { bicycle parking. IAD } 3\end{array}$ & $\begin{array}{l}\text { Car-free city. Underground } \\
\text { Urban Transit to free public } \\
\text { space. IAD } 4\end{array}$ \\
\hline $\begin{array}{c}\text { Transportation Demand } \\
\text { Management }\end{array}$ & $\begin{array}{l}\text { Promotes vehicle sharing. } \\
\text { IAD } 0\end{array}$ & $\begin{array}{c}\text { Use of Urban Transit System. } \\
\text { IAD } 4\end{array}$ \\
\hline $\begin{array}{c}\text { Access to Civic and Public } \\
\text { Spaces }\end{array}$ & $\begin{array}{l}\text { Public space inside the block. } \\
\text { IAD } 3\end{array}$ & $\begin{array}{c}\text { Due to the underground } \\
\text { characteristic of the transit } \\
\text { system, most public space } \\
\text { will be passive. IAD } 4\end{array}$ \\
\hline $\begin{array}{c}\text { Access to Recreation } \\
\text { Facilities } \\
\end{array}$ & $\begin{array}{l}\text { Recreational facilities within } \\
\text { the block. IAD } 3\end{array}$ & NINF \\
\hline $\begin{array}{l}\text { Visitability and Universal } \\
\text { Design }\end{array}$ & $\begin{array}{l}\text { Several types of dwelling } \\
\text { units /concern with mobility. } \\
\text { IAD } 3 \\
\end{array}$ & NINF \\
\hline $\begin{array}{l}\text { Community Outreach and } \\
\text { Involvement }\end{array}$ & $\begin{array}{l}\text { Calls for an holistic design } \\
\text { approach. IAD } 0\end{array}$ & NINF \\
\hline Local Food Production & $\begin{array}{c}\text { Includes local food } \\
\text { production on the rooftops. } \\
\text { IAD } 4\end{array}$ & $\begin{array}{c}\text { Includes local food } \\
\text { production outside city } \\
\text { perimeter IAD } 3\end{array}$ \\
\hline $\begin{array}{l}\text { Tree-Lined and Shaded } \\
\text { Streets }\end{array}$ & Tree-lined streets. IAD 3 & $\begin{array}{l}\text { Main streets are tree-lined; } \\
\text { other streets are shaded and }\end{array}$ \\
\hline
\end{tabular}


narrow. IAD 3

\begin{tabular}{|c|c|c|}
\hline $\begin{array}{c}\text { ND-Green Infrastructure + } \\
\text { Buildings }\end{array}$ & & \\
\hline Certified Green Building & Goal:LEED Platinum. IAD0 & Goal= Carbon Neutral IAD 0 \\
\hline Water-Efficient Landscaping & $(=$ LEED NC IAD 4) & $(=$ LEED NC IAD4) \\
\hline $\begin{array}{l}\text { Minimized Site Disturbance } \\
\text { in Design and Construction }\end{array}$ & NINF & $\begin{array}{l}\text { Mitigation actions predicted. } \\
\text { IAD } 0\end{array}$ \\
\hline Heat Island Reduction & (= LEED NC. IAD 4) & (= LEED NC. IAD 4) \\
\hline Solar Orientation & $\begin{array}{l}\text { East-west axis 5xlonger than } \\
\text { north-south axis. Block } \\
\text { orientation greater than } 15^{\circ} \\
\text { from the geographic east- } \\
\text { west. IAD } 3\end{array}$ & $\begin{array}{c}\text { Narrow streets and compact } \\
\text { design. IAD } 3\end{array}$ \\
\hline $\begin{array}{l}\text { On-Site Renewable Energy } \\
\text { Sources }\end{array}$ & $\begin{array}{l}\text { On the rooftop - Solar } \\
\text { thermal energy }+ \\
\text { photovoltaic + wind energy. } \\
\text { Roof design expands surface } \\
\text { to maximize production. } \\
\text { Solar cells on the } \\
\text { southwestern façade. IAD } 4\end{array}$ & $\begin{array}{l}\text { On the rooftop - almost } 80 \% \\
\text { of the consumed energy will } \\
\text { be solar. The entire city } \\
\text { covered with panels. IAD } 4\end{array}$ \\
\hline $\begin{array}{l}\text { Solid Waste Management } \\
\text { Infrastructure }\end{array}$ & $\begin{array}{c}\text { Organic waste used as } \\
\text { fertilizer (predicted). IAD } 0\end{array}$ & $\begin{array}{l}\text { Organic waste used as } \\
\text { fertilizer (predicted). IAD } 0\end{array}$ \\
\hline
\end{tabular}

\section{Results}

Two types of results can derive from Table 1. The first result is that all items classified as IAD 4 (with one exception) regard either the use of rooftops or the planned transport system (e.g. Table 1). The second result is a high frequency of strategies classified as IAD 3, followed by IAD 4 and IAD 0, with only a few items classified as IAD 1 or 2 (e.g. Fig.1).

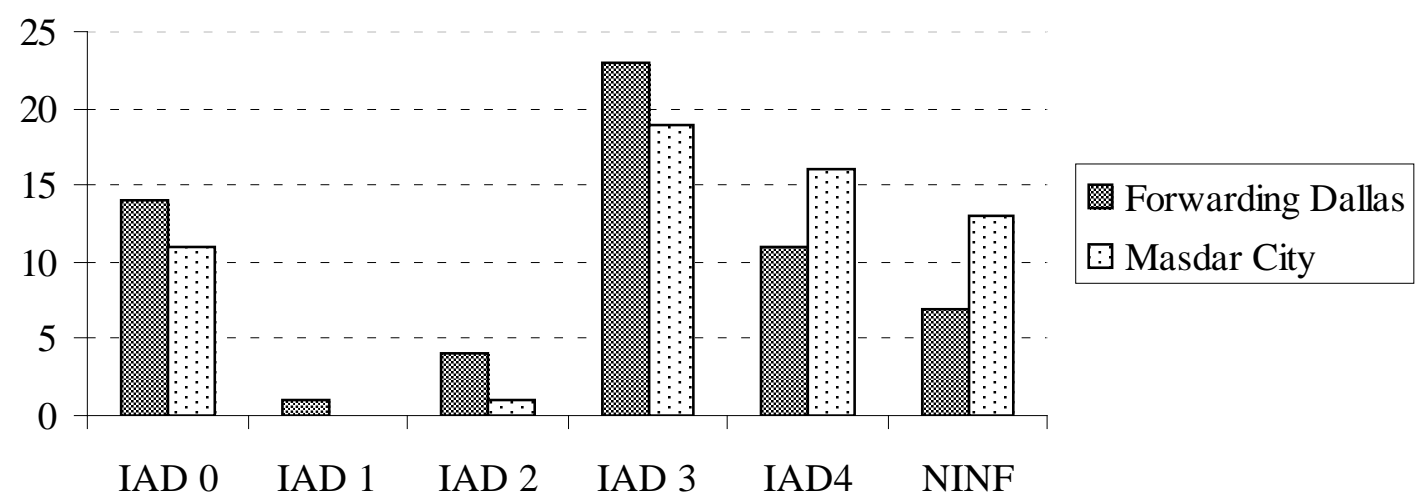

Fig. 1 Frequency of IAD (Impact on Architectural Design) according to classes (IAD 0 to $I A D$ 4)

\section{Discussion and Conclusions}

Based on these results, we can argue three conclusions. The first conclusion is that two new technologies which might become a trademark of the city of the future are: the increased relevance of the rooftop as a "productive surface", with the potential to collect energy and rainwater, and to provide spaces for plantation; and the inclusion of intelligent urban transport systems, allowing public space to be car-free. This conclusion is based on the indication that 
almost all strategies classified as IAD 4 are related either to rooftops or to the urban transport system. The second conclusion derives from the low frequency of IAD 1 and IAD 2, indicating that fewer design strategies are related to the surface of the architectural object (or to the architectural skin) as compared to the configuration of the architectural from. Most strategies used by the selected practitioners to comply with the LEED categories fall under IAD 0, IAD 3 or IAD 4; hence, there is either no impact on architectural design (IAD 0) or a profound impact on the configuration of architecture (IAD 3 and IAD 4). The third conclusion derives from the high frequency of strategies classified as IAD 3 (e.g. Fig.1), indicating that the selected practitioners are using existing or traditional design strategies to relate architecture effectively to climate and location.

What we believe to be particularly interesting about these results is that the first conclusion has the potential of becoming global in its application as practitioners all over the world (literally) turn their projects to the sun and build intelligent urban transport systems, while the third conclusion indicates a promotion of local characteristics; hence, the carbon neutral city of the future might present an architectural language which incorporates both global and local design characteristics, a language where tradition meets technology to achieve a most efficient architectural design.

\section{References}

[1] US Green Building Council, LEED 2009 for New Constructions and Major Renovations Rating System, 2009, pp. 1-88.

[2] Congress of New Urbanism, Natural Resources Defence Council and US Green Building Council, LEED 2009 for Neighborhood Development Rating System, 2009, pp. 1-122.

[3] MOOV+DATA, "Forwarding Dallas”, Technical drawings, Project Description and interviews conducted by the authors, 2010.

[4] Reiche, D., Renewable Energy Policies in the Gulf Countries: a case-study of the Carbon Neutral "Masdar City" in Abu Dabhi, Energy Policy, 2009, doi: 101016 / j.enpol.2009.09.28

[5] Masdar City, Abu Dhabi Future Energy Company, www.masdar.ae

[6] Foster+Partners, www.fosterandpartners.com.

[7] Habitat Futura, Masdar, La Ciudad del Futuro, n.24, 2010, pp. 37-46.

[8] Nader, S., Paths to a low-carbon economy - the Masdar Example, Energy Procedia 1 (2009) doi:10.1016/j.egypro.2009.02.99

[9] Boyer,JL et. al., Systems integration for cost effective Carbon Neutral Buildings: A Masdar headquarters case-study, Proceedings of the 4TH ASME International Conference on Energy and Sustainability, Vol.1, 2010.

[10]Roseta V.M., F. "From Critical to Carbon Neutral, Grassroots Sustainable Urban Planning” in C.A. Brebbia, et al (ed.) The Sustainable City VI - Urban Regeneration and Sustainability, Southampton: WIT Press, 2010, pp. 629/636.

[11]Hyder Consulting, “Environmental Impact Assessment Report”, Masdar Delivery Phase 1, April 2009, pp. i-xiv. 